EOMmunutriln? Communication et organisation

Or

Le coaching, enjeux, paradoxes et perspectives

\title{
Eugène Enriquez
}

\section{(2) OpenEdition \\ 12 Journals}

Édition électronique

URL : http://journals.openedition.org/communicationorganisation/3357

DOI : 10.4000/communicationorganisation.3357

ISSN : $1775-3546$

Éditeur

Presses universitaires de Bordeaux

Édition imprimée

Date de publication : 1 janvier 2006

ISSN : 1168-5549

Référence électronique

«Eugène Enriquez », Communication et organisation [En ligne], 28 | 2006, mis en ligne le 19 juin 2012, consulté le 30 avril 2019. URL : http://journals.openedition.org/communicationorganisation/3357 ;

DOI : 10.4000/communicationorganisation.3357

Ce document a été généré automatiquement le 30 avril 2019.

(C) Presses universitaires de Bordeaux 


\section{Eugène Enriquez}

\section{NOTE DE L'ÉDITEUR}

Avec Françoise Perdriset

1 Eugène Enriquez est Professeur émérite de Sociologie à l'Université Paris 7-Denis Diderot, Consultant auprès d'organisations en France et à l'étranger.

2 Co-fondateur de l'ARIP, Association pour la Recherche et l'Intervention Psychosociologique, 1959 ;

3 Co-fondateur, en 1972, de la revue Connexions, et rédacteur en chef jusqu'en 1991 ;

4 Co-fondateur du CIRFIP, Centre International de Recherche, d'Intervention et de Formation Psychosociologique, en 1993 ;

5 Co-fondateur de la Revue Internationale de Psychosociologie, en 1993, et co-rédacteur en chef jusqu'en 2005 ;

6 Co-fondateur de la Nouvelle Revue de Psychosociologie (premier numéro à paraitre aux éditions Érès) ;

7 Co-directeur, avec Vincent de Gaulejac, du Laboratoire de Changement Social de L'Université Paris 7, jusqu'en 1997 ;

8 Membre fondateur de l'ISPSO, International Society for the Psychoanalytic Study of Organizations, en 1985.

Monsieur Enriquez, vous avez consacré l'essentiel de vos travaux, dès les années cinquante, à l'étude des organisations, ce qui a fait de vous un pionnier de l'observation de leurs différents modes de fonctionnement. À ce moment-là, qu'est-ce qui vous a conduit vers ce choix?

Je suis un enfant de la deuxième guerre mondiale, non pas que je l'ai vécue de façon terrifiante, puisque je vivais en Tunisie et que ce pays n'a été occupé par les Allemands que pendant six mois. J'avais des parents très politisés, j'étais au courant de tout, je devais avoir onze, douze ans. On écoutait la $B B C$, on savait ce qui se passait et on prévoyait ce qui pouvait se passer. J'avais un oncle qui se cachait, mon frère qui se 
cachait, mon père a failli être déporté. Et je me suis demandé, dès cette époque, pourquoi des nations aussi civilisées pouvaient être susceptibles d'autant de barbarie.

La problématique "barbarie et civilisation " a toujours été extrêmement importante pour moi, ce qui fait que pendant un certain temps j'ai eu envie d'agir au niveau politique. J'ai commencé à faire mes études et je voulais, non pas devenir un homme politique, mais influencer le jeu politique. Le temps a passé et je me suis rendu compte que je ne serais jamais un grand fonctionnaire public et, en particulier, ce que je pensais être un diplomate parce que je manquais de diplomatie et que j'avais envie de dire véritablement tout le temps ce que je pensais. Donc je me suis reposé une autre question. Comment néanmoins avoir une action qui ait un intérêt pour les citoyens, un intérêt humain, social?

À ce moment-là, la problématique et les méthodes de la psychosociologie commençaient à être importées des États-Unis, ce qui m'a permis de participer au premier séminaire de dynamique de groupe fait avec des disciples de Kurt Lewin. Très rapidement avec d'autres, nous nous sommes lancés dans cette aventure psychosociologique. Ce qui nous plaisait, c'était l'idée que l'on pouvait aider les gens à devenir plus démocrates dans la vie concrète et non pas sur le plan théorique ou sur le plan des idées générales. L'effort de Lewin et de ses collaborateurs avait été, en inventant la dynamique de groupe, de faire en sorte que les gens se posent des questions concernant les communications interpersonnelles, la reconnaissance de l'altérité et la possibilité de la décision collective. Dans un petit bouquin que j'avais écrit en 1971, en collaboration avec Goguelin et Dubost, sur La formation psychosociale dans les organisations ${ }^{1}$ je montrais que ce qui se jouait dans la formation était un apprentissage de la démocratie.

\section{Et dans les organisations?}

$\mathrm{Au}$ début, nous pensions que si l'on prenait, dans des séminaires de dynamique de groupe, des responsables d'organisations et s'ils arrivaient à changer profondément, une fois rentrés dans leurs organisations, ils y apporteraient d'autres méthodes de commandement, de compréhension des gens, de communication. C'est, par exemple, ce que nous avons développé avec Palmade et Dubost au sein des séminaires que nous faisions à EDF. Et puis, petit à petit, on s'est rendu compte, Guy Palmade, Max Pagès, André Lévy, Jean-Claude Rouchy, Jean Maisonneuve et moi, que l'organisation c'est quand même un peu plus que le groupe, qu'il y avait, dans le groupe, de la relation spontanée mais pas de formalisation. Si quelqu'un dans un groupe a changé profondément, s'il veut essayer de changer les choses lorsqu'il revient dans son organisation, il va rencontrer des résistances parce que les autres n'ont pas changé. Ce qui a amené très vite l'idée qu'il fallait faire des interventions dans les organisations et que la psychosociologie n'avait pas seulement pour champ le groupe, mais aussi l'organisation et l'institution. Nous nous sommes aperçus rapidement que les Américains et les Anglais nous avaient montré la voie, mais qu'il y avait un côté un peu idyllique, gentil dans la démarche américaine. Influencés par la psychanalyse, nous nous sommes dit que les êtres humains, malgré tout, ce n'était pas si simple que cela, qu'à côté des pulsions de vie, il existait aussi des pulsions de mort, ce à quoi je crois de plus en plus, et que les êtres humains, les sociétés étaient des mélanges, subtils et complexes, de tendances à l'amour, à l'amitié, à la fraternité, et des tendances à la destruction, à la haine de l'autre, à la bataille et à la compétition. 
Cela nous a amenés à introduire, en particulier dans mon cas, des références à la théorie psychanalytique. C'est-à-dire, d'examiner s'il y avait des processus inconscients de groupe qui traversaient l'organisation, s'il y avait des peurs, des angoisses, sans pour cela plaquer totalement la théorie psychanalytique sur les organisations. Dans la préface de mon livre L'organisation en analyse ${ }^{2}$,je dis qu'il ne s'agit pas d'expliquer tout le fonctionnement organisationnel à partir de la théorie psychanalytique. Je ne suis pas dans l'optique "psychanalyse et management». La psychanalyse peut nous apporter une certaine approche mais il y a aussi, dans le travail en groupe, dans celui des organisations, des phénomènes parfaitement rationnels et volontaires, que l'on doit accepter. D'autant plus que, si je reprends ce que je disais sur la deuxième guerre mondiale, j'avais été très frappé par les phénomènes politiques et, quand on dit les phénomènes politiques, on dit aussi les phénomènes de politique d'entreprise, de politique d'organisation, de normes sociales. Cela ne peut pas être totalement travaillé par la théorie psychanalytique à moins de tout réduire, or j'ai toujours été contre toutes les tendances réductionnistes. Il faut avoir une approche transdisciplinaire, en essayant des concepts qui viennent d'un certain lieu mais qui peuvent être appliqués, plus ou moins transformés, dans d'autres lieux. Le concept d'anomie, qui sert à désigner les moments de décomposition, les pertes de références, qui vient de la sociologie, peut être appliqué au petit groupe et à l'individu. De la même manière, le terme de refoulement vient de la psychanalyse; eh bien, il peut y avoir du refoulement organisationnel, du refoulement social.

Fondateur de la psychosociologie analytique, vous avez voué toute une carrière à cette discipline. Quelle définition en proposez-vous actuellement?

Je ne me considère pas comme le fondateur. Quand on a créé notre première association, l'ARIP, l'Association pour la Recherche et l'Intervention Psychosociologique, je jouais un rôle déjà important mais les deux véritables fondateurs ont été Guy Palmade et Max Pagès. Il y avait déjà des références psychanalytiques et c'est moi qui ai peut-être poussé le plus dans cette voie. Pour comprendre quelque chose sur moi, je crois qu'il faut toujours revenir à la question que je me suis posée au départ, sur barbarie et civilisation. J'ai essayé de traiter cela dans un certain nombre de textes, dans De la horde à l'État ${ }^{3}$, la face obscure des démocraties modernes ${ }^{4}$,etc. Par exemple, le nazisme n'a pas été simplement un fascisme comme chez Mussolini, Franco et Salazar. Chez Hitler, il y avait l'obsession de la destruction des Juifs, des Tziganes, des malades mentaux, des autres... Cette idée de pureté, tellement forte, qu'il devait se débarrasser de toutes les souillures possibles, fait qu'on ne peut pas comprendre simplement cela par l'économie de l'Allemagne et les conditions socio-historiques, à ce moment-là. Il y avait une volonté mortifère extraordinairement importante. Donc je me suis continuellement demandé si, dans les organisations, les institutions, les individus, il ne faut pas aussi tenir compte de cette part inconsciente, bizarre qui peut engendrer les conséquences les meilleures comme les plus catastrophiques mais dont on ne fait pas suffisamment état. Et c'est pour cela que la psychanalyse a toujours été, pour moi, un élément important de compréhension. Sauf qu'à certains moments, je m'aperçois que les gens avec qui je travaille ne peuvent pas intégrer une telle optique.

Ma perspective psychosociologique de recherche-action a toujours été de travailler avec les personnes, les groupes, les organisations en voyant jusqu'où ils peuvent aller, de façon à les accompagner. J'avais utilisé ce terme d'accompagnement dans les années 1972, de manière à les accompagner dans leur développement, dans leur interrogation 
et non pas en essayant de les forcer. C'est une des choses qui a fait que pendant un certain temps, nous nous sommes beaucoup bagarré avec les tenants de l'analyse institutionnelle, Georges Lapassade, René Lourau. Si comme dans l'analyse institutionnelle, on dit que la seule démocratie possible est l'autogestion, eh bien on ne peut pas imposer l'autogestion, c'est totalement contradictoire dans les termes. En plus de cela, je n'utilise jamais de référence explicite à des concepts psychanalytiques. Tout ce que l'on peut faire c'est de faire percevoir aux gens qu'il y a peut-être des dimensions oubliées qui ont de l'influence. Donc c'est, comme disait la psychanalyste Piera Aulagnier, une libre théorisation flottante qui est présente dans mon esprit. Ce qui est important c'est le type de processus dans lequel les gens sont et la capacité d'accompagnement et d'écoute véritable que l'on a. Cela ne veut pas dire qu'à un certain moment on ne puisse pas provoquer un petit peu les gens, leur montrer les contradictions dans lesquelles ils sont. Il y a une exigence interprétative, mais il faut respecter la capacité des gens à pouvoir s'approprier les interprétations et il ne faut pas que les interprétations proposées soient faites tout simplement pour vous ébranler. Il s'agit seulement qu'elles puissent être des éléments importants dans le processus d'évolution.

Donc je veux bien qu'on dise, pour caractériser ma démarche, psychosociologie analytique, parce que c'est plus facile. On me met là et je ne suis pas contre, en même temps, je suis un des fondateurs, avec Vincent de Gaulejac et les autres, de la sociologie clinique, je suis un des premiers aussi à m'être occupé de la sociologie des organisations et dans certains pays je trouve que je suis considéré, à tort ou à raison, comme un des papes de la psychosociologie des organisations. Je n'ai pas envie de me laisser enfermer, ce que je peux dire de façon générale c'est que je suis un psychosociologue ouvert à la sociologie, ouvert aux phénomènes socio-économiques, aux phénomènes sociohistoriques et particulièrement intéressé par la théorie psychanalytique, par le fait que pour moi les pulsions, les grands phénomènes de refoulement, d'idéalisation et de sublimation existent non seulement dans l'individu mais aussi dans le champ social.

Vous avez introduit la psychosociologie dans les entreprises comme méthode de distanciation. Que s'est il passé dans les entreprises à ce moment-là ? Qu'est-ce que vous observiez?

Ce n'est pas spécialement de la distanciation. C'est le fait de ne pas se poser comme organisateur conseil, conseil en organisation mais comme des consultants. D'ailleurs le terme de consultant est devenu un terme à la mode. Je crois que c'est notre première équipe qui, dans les années 1955-1960, l'a lancé. Qu'est-ce que cela veut dire, être consultant psychosociologue? C'est aider les entreprises à bien poser leurs problèmes, à reconnaître les problèmes qu'elles ont, que ce soit des problèmes relationnels, communicationnels, ou de structure. Et petit à petit, travailler dans un processus plus ou moins long, qui permet aux gens de co-construire avec vous le type de solution, et non pas d'intervenir comme un expert qui, après avoir fait le diagnostic, donne un certain nombre de conseils. Notre pensée a toujours été de reconnaitre profondément l'altérité des gens et de se dire que ce sont eux qui en bénéficieront ou qui en paieront les pots cassés. Il faut se rendre compte de la responsabilité que l'on a, il faut aller avec les gens aussi loin qu'ils peuvent aller et on ne peut pas les forcer à aller plus loin dans leur manière d'identifier les problèmes, de les traiter, de transformer leur structure, leurs relations. Il faut avoir un respect, je ne dirais pas inconditionnel comme disait Rogers, c'est un peu exagéré, mais il faut avoir le respect des gens avec qui on travaille. 
Naturellement, il peut y avoir des groupes antagonistes et il faut faire en sorte d'être justement suffisamment distancié pour ne pas prendre parti pour un groupe par rapport à un autre, et travailler de façon à ce qu'ils puissent progressivement, au travers des analyses qu'on les aide à faire, entrer dans des processus de facilitation, de coopération plutôt que de compétition ou de rivalité.

C'était l'objectif de vos interventions?

Oui. Les gens coopèrent plus volontiers parce qu'ils ont moins peur les uns des autres, ils ont moins de préjugés, ils rentrent dans de meilleures communications, ils identifient de façon commune leurs problèmes et ils s'aperçoivent qu'ils ont plus d'intérêts communs qu'ils ne le pensaient au départ. Ceci étant, il faut être honnête, des fois cela marche, des fois cela ne marche pas. On se trouve parfois à devoir faire avec des attitudes de rivalité, d'antipathie, de violence tellement fortes que, malgré tout ce travail progressif, on s'aperçoit que les gens ne veulent pas nous écouter et qu'il y a des blocages, pour des raisons tenant à nous-mêmes ou aux autres ou à cette dynamique entre nous-mêmes et les autres, et s'ils ne peuvent pas être levés, il y a échec.

Et il faut aussi bien dire la vérité, les entreprises appelaient les psychosociologues beaucoup plus dans le temps que maintenant. Les entreprises n'étaient pas obsédées comme elles le sont à l'heure actuelle par la survie, le fait de dégager la meilleure valeur possible pour satisfaire les actionnaires. C'étaient des entreprises dirigées soit par des propriétaires, soit par des managers compétents. En plus de cela, c'était le plein emploi, les entreprises se développaient. Si on prend des interventions qui ont été faites, en particulier, par Max Pages, par notre équipe de l'ARIP, on s'aperçoit que les gens disaient : «On se porte très bien, simplement on veut se porter encore mieux, si vous nous aidez, on est prêt d'ailleurs à y mettre du nôtre, à s'interroger profondément, même la direction générale est prête à s'interroger, à se remettre en question. Mais si on s'aperçoit que tout cela c'est de la frime, eh bien, on vous dit au revoir ». Maintenant, quand les entreprises font appel, elles sont plus en état de crise et c'est beaucoup plus difficile. Elles ont plus tendance à appeler des gens qui les rassurent très vite que des personnes avec qui il y a un long travail à faire. Cela ne veut pas dire qu'il n'y en ait pas, vous pouvez vous référer au beau livre écrit par mes collègues Jacqueline Barus-Michel et Florence Giust-Desprairies qui montre des interventions dans des situations de crises profondes ${ }^{5}$.

À l'heure actuelle, les demandes viennent beaucoup plus souvent du secteur sanitaire et social que des entreprises. Je dis toujours que la psychosociologie est née dans le petit groupe et dans l'entreprise, et qu'aujourd'hui, elle se développe beaucoup plus dans des secteurs où le problème humain est pris à bras le corps. Dans le secteur sanitaire et social, on ne peut pas ne pas le traiter comme dans les entreprises qui sont devenues globalement beaucoup plus compétitives, beaucoup moins relations humaines, beaucoup plus relations inhumaines.

D'après votre expérience, comment vous représentez-vous les entreprises hypermodernes?

J'en connais un certain nombre, moins que Nicole Aubert et Vincent de Gaulejac parce que je suis maintenant beaucoup plus âgé qu'eux et que j'interviens moins. À l'heure actuelle, dans l'entreprise, disons hypermoderne, on peut faire des recherches. Nicole Aubert et Vincent de Gaulejac en parlent, ils ont pu faire des analyses, ils n'ont pas fait d'interventions. On a accepté qu'ils aillent interroger, on n'a pas accepté qu'ils essayent 
de changer quoi que ce soit. Ma position particulière, est que, en dehors des recherches historiques, des recherches pour affiner des concepts, je ne m'intéresse à la recherche que si elle peut aboutir à une intervention. Toute recherche est déjà, dans une certaine mesure, une intervention, puisque les gens se mettent à parler des problèmes et que très souvent, ils évoquent des problèmes dont ils n'avaient pas forcément conscience. Cela me gêne toujours d'écouter des gens, au besoin de leur dire ce qu'il en est, ce qui est normal, mais d'avoir peut-être suscité des désirs, des espoirs en parlant de tout cela et de leur dire non, il n'y aura pas de changement, il ne s'agit que de recherche. Je ne dis pas que cela ne soit pas possible, que les gens qui font cela ne sont pas parfaitement honnêtes, au contraire. Pour ma part, je n'ai jamais pu faire de recherche qui ne soit pas directement appliquée dans une intervention. Je pense qu'il y a suffisamment de très bons chercheurs en France qui me fournissent plein d'éléments. Je n'interviens pas dans une entreprise hypermoderne à l'heure actuelle, sauf quand elle m'appelle pour faire une conférence dans laquelle je dis honnêtement ce que je pense, les membres de l'entreprise m'écoutent gentiment et puis après quoi je disparais.

Si je reprends ce que disent mes collègues, Vincent de Gaulejac sur La société malade de la gestion $^{6}$, ou Nicole Aubert sur Le culte de l'urgence ${ }^{7}$, je constate que faire un travail de longue haleine, en instaurant des processus de travail, en montrant que le problème humain n'est pas simplement un problème de ressources humaines, terme que je déteste, mais un problème de ce qui est le plus vif et le plus dynamique, devient de plus en plus difficile dans ce type d'entreprise. L'entreprise hypermoderne me semble être née sous le signe de la compétitivité, soit de l'individualisme, soit du petit groupe en rivalité avec les autres, dans une espèce d'émulation continue de toujours plus, de toujours mieux, de l'obsession de comment améliorer les choses et non pas de pourquoi améliorer les choses. Je suis un homme du pourquoi avant d'être un homme du comment. J'espère que les gens qui interviennent plus dans ces entreprises hypermodernes tiennent compte de ce que j'évoque mais, si je ne trouve pas les conditions minima pour qu'un certain type de travail puisse se faire, je ne le fais pas. D'ailleurs les entreprises ne me le demanderont pas, elles sont intéressées uniquement par leur réalité immédiate.

Depuis toujours, vos travaux s'inscrivent dans la problématique d'un pouvoir des organisations s'exerçant à l'encontre du désir de la personne au travail. Vous opposez à la rationalisation, qui serait une perversion de la raison, une vérité polymorphe. Comment se fait-il que l'individu, dont on n'a jamais autant parlé, est aussi souffrant?

Je me suis toujours intéressé au phénomène du pouvoir parce que cela me semble être un élément organisateur central de toutes les organisations, comme de la société. On ne peut pas ne pas s'interroger continuellement sur les problèmes de pouvoir, sur les problèmes d'autorité. Qu'est-ce qui se passe à l'heure actuelle? Pourquoi l'individu dont on n'a jamais autant parlé est-il aussi souffrant? Je dirais que c'est justement parce qu'on ne parle que de lui. Je me méfie toujours quand on écrit l'humain avec un grand $\mathrm{H}$. Plus on parle de l'humain, (je dis que c'est une loi quasiment sociologique, même s'il n'y a guère que moi qui l'ai énoncée), plus on élève l'individu dans le discours, plus on le rabaisse dans les actes. Je prends un exemple monumental, que je donne souvent: c'est quand même Staline qui a dit, le premier, que l'homme était le capital le plus précieux et on voit ce qu'il en a fait. Quand on est tellement centré sur l'homme, sur l'individu qui doit devenir autonome, prendre des décisions, des initiatives, qu'est-ce que l'on fait? On finit par le rendre responsable de tout. C'est 
effectivement un des problèmes de la psychologisation des difficultés à l'heure actuelle, alors que bien des problèmes sont liés à la conjoncture, à la politique de l'entreprise, à ses modes d'organisation et non pas simplement à ce que fait tel ou tel individu. Je ne suis pas le seul à évoquer tout cela. Richard Sennett, il y a déjà quinze ans aux ÉtatUnis, avait dit que le moi de chaque individu devenait un fardeau de plus en plus lourd. Alain Ehrenberg a écrit un livre sur la fatigue d'être soi ${ }^{8}$. Le fait de se dire que l'on perd tous les points de repère, que le seul point de repère c'est soi-même, finit par être étouffant, d'autant plus que dans l'entreprise on renforce tout cela. Les gens sont continuellement stressés parce que de plus en plus l'entreprise est une course d'obstacles. Comme dans le sport, il faut toujours se dépasser, à chaque moment, c'est une épreuve. Ils sont terrifiés à l'idée de faire des erreurs. La réussite est très peu mise à leur crédit, l'échec est mis immédiatement à leur débit. Vous ajoutez les cadences, la flexibilité du travail... Tout le monde sait qu'on peut disparaitre du jour au lendemain même si on a été fidèle, loyal, s'il y a une OPA, une fusion. Dans une certaine mesure, tout le monde est menacé. C'est justement là que se situe aussi le fameux coaching.

Précisément, dans ce numéro de Communication et Organisation, le dossier tente d'explorer le coaching dans sa dimension pratique et événementielle. Spécialiste de la psychosociologie, quel regard portez-vous sur l'apparition du coaching, sur son développement et sur ses enjeux stratégiques?

Il me semble que c'est une apparition, une invention de cette société de l'individualisme, parce que les gens sont tellement sollicités pour réussir. On se rend compte que certains ont des potentialités. Il faut pouvoir les utiliser, on ne peut pas employer tout le monde pour immédiatement être quelqu'un d'autre, donc il faut les aider, mais en essayant de faire en sorte de bien les aider. Je n'ai rien contre le coaching si on le prend comme un accompagnement des individus pour qu'ils puissent se développer eux-mêmes; je suis plus interrogatif si c'est le type de développement uniquement désiré par l'entreprise. À ce moment-là, cela me semble être une espèce de formatage déguisé de l'individu. Je ne suis donc pas contre, je serais encore moins contre si effectivement le coaching pouvait se développer vis-à-vis d'autres groupes, d'autres individus que les cadres supérieurs. Après tout, tout le monde peut avoir besoin d'être aidé. Mais je pense que c'est une espèce de palliatif qui est donné à cette société. Et je serais très inquiet si le succès du coaching faisait disparaître toutes les possibilités de recherche-action et d'interventions psychosociologiques, parce que cela voudrait dire que l'on se centre totalement sur l'individu non pas sur l'organisation dans son ensemble, que l'on renforce cette idée que chacun doit être le meilleur possible. Or, quand même! On sait bien que l'on ne peut pas, premièrement être toujours le meilleur possible, deuxièmement être bon dépend aussi des structures, de l'environnement, des conditions dans lesquelles vous pouvez effectivement vous développer. Or cette relation individu-organisation est en train de disparaître. Donc je suis quelque peu interrogatif sur le coaching et sur ses possibilités de développement. Ceci étant, la publication de la Société Française de Coaching me semble intéressante dans la mesure où effectivement cela donne vraiment l'impression que les coachs individuels sont de plus en plus conscients de l'ensemble des questions qui se posent.

Vous parlez de sa publication sur la démarche déontologique?

Oui, les coachs sont de plus en plus conscients de cela. Mais je m'interroge, malgré tout, toujours sur cette idée d'accompagnement personnalisé comme si la personne n'était 
pas dans un groupe, dans une structure, avec des problèmes dont elle n'a pas forcément la maîtrise.

Les coachs et les psychosociologues parlent d'accompagnement. N'y a-t-il pas des risques de confusion ? Pourrait-on les croire apparentés ?

Oui, il peut y avoir des confusions; d'ailleurs c'est pour cela que j'ai parlé d'accompagner les gens, mais je ne le dis pas, je ne mets pas la psychosociologie comme une fonction d'accompagnement, je la mets comme une action de changement, de changement social, voulu, en collaboration avec les autres.

Le vocabulaire de la psychosociologie, qui était spécifique à la psychosociologie, a été totalement pillé par les économistes, par les gestionnaires et maintenant par les gens du coaching. La motivation, le changement, l'évolution, le développement personnel... C'est comme cela, nous faisons notre travail. Si nous sommes reconnus tant mieux, si nous ne le sommes pas tant pis! Vous savez c'est une ligne sage. Dans un de mes vieux textes, je disais aussi qu'il fallait avoir de l'humour, de la frivolité, de l'ironie. Je le pense toujours, je suis pour des psychosociologues souriants et non pas pour des consultants graves, sérieux et ennuyeux. Alors les psychosociologues font toujours de la recherche-action, de l'intervention même si le terme n'est pas très bon, c'est un terme un peu violent. En plus de cela, la grosse différence, c'est que les psychosociologues n'accompagnent jamais d'individus. Ils accompagnent des groupes, des organisations, et ils ne se transforment pas en psychothérapeutes individuels. Il y a souvent plus de rapport entre le coaching et une thérapie légère de soutien individuel, qu'entre le coaching et la psychosociologie.

Je reste, je suis un psychosociologue pur, si l'on peut s'exprimer ainsi. J'ai toujours désiré, dans les groupes de formation, de perfectionnement ou d'évolution, avoir des gens qui viennent ayant décidé par eux-mêmes de venir. Quand j'interviens dans des organisations, je demande toujours, après avoir expliqué ce qu'il en est et après avoir eu souvent des discussions avec les syndicats, si les gens sont volontaires pour suivre le travail et s'ils décident de ne pas venir, je ne cherche pas à leur faire changer d'avis. L'intérêt que j'ai toujours porté au problème du pouvoir a été, non pas de faire en sorte que les gens sachent mieux assurer leur pouvoir sur les autres, mais de faire en sorte que chacun puisse exercer le minimum de pouvoir qu'il est capable de développer en lui. Cela va dans une perspective d'autonomisation des individus. Je suis très proche de la pensée de Castoriadis, à ce point de vue-là. C'est permettre aux gens, auxquels on a souvent refusé le droit à la parole et au pouvoir, de pouvoir prendre la parole, d'utiliser le minimum de pouvoir dont chacun peut disposer. Cette attitude est peut-être empreinte d'idéologie, mais je reste fondamentalement lié à un idéal démocratique véritable. Essayons de faire que le plus grand nombre d'individus puisse devenir le plus autonome possible et puisse travailler avec d'autres, dans des collectifs dans lesquels l'invention et la créativité sont possibles. Je n'ai jamais caché le fait de vouloir une civilisation non barbare, de vouloir une civilisation démocratique, c'est pour cela que mes premières impressions d'enfance ont été pour moi totalement déterminantes, dans la manière dont je me suis conduit et dont je me conduis encore maintenant.

Je dirais en plus que les évolutions personnelles dans des groupes qui existent sont le fruit du travail en groupe et de sa répercussion sur chacun des individus. Chacun en a fait son miel mais le miel de l'un n'est pas forcément le miel de l'autre. La personne arrive à mieux se situer dans ses responsabilités par rapport à son travail, par une 
meilleure compréhension des phénomènes organisationnels. Mon idée a toujours été d'aider les gens à lier le développement personnel avec un développement organisationnel.

Les études faites sur le commandement, sur les aptitudes, sur le leadership, qui reviennent à la mode, vont tout à fait dans le même sens que l'idée du coaching. On dit que le leadership est le pivot de l'organisation, mais pas du tout! Si la division du travail est mal faite, si les valeurs sont inexistantes, ou contradictoires ou encore relevant plus d'une éthologie que d'une éthique, si le produit ne vaut rien, on peut avoir tout le leadership que l'on veut, cela ne sert à rien du tout. Pour moi, l'être humain ne peut s'exprimer que par rapport au contexte. Il y a des contextes dans lesquels les gens sont étouffés, des contextes dans lesquels les gens s'épanouissent, et donc faire de la psychologisation des problèmes m'a toujours semblé quelque chose d'absolument ridicule, c'est faire reporter sur les épaules de quelqu'un tout un fonctionnement social.

En conclusion de votre ouvrage Les jeux du pouvoir et du désir dans l'entreprise ${ }^{9}$ vous écrivez: «L'entreprise et la société moderne ont tout essayé pour rendre les gens passifs et conformistes. Alors que leur intérêt bien géré est d'avoir, en leur sein, des sujets capables de leur insuffler leur dynamisme. Elles ont tout essayé. Elles n'ont réussi que partiellement. Partout la résistance s'organise ». Comment cette résistance se manifeste-t-elle?

Pour le moment je ne crois pas à beaucoup de résistance. En fait, il y a deux tendances. À un certain moment, il y avait bien cette idée d'idéalisation, de passion de l'entreprise et maintenant, comme je le disais tout à l'heure, les gens s'aperçoivent que même s'ils sont fidèles, loyaux, soumis, conformes et en même temps dynamiques, pleins d'initiative, la loi du marché peut faire qu'ils soient mis à la porte. Il y a une espèce de retrait des gens.

D'un autre côté, pour ceux qui ont des diplômes, qui se sentent plus sûrs d'eux-mêmes, il y a le développement d'une mentalité de mercenaire : "Je suis dans telle entreprise parce qu'elle me paye bien, elle me donne des possibilités, mais si je trouve un chasseur de têtes, je m'en vais sans le moindre problème ». Alors que, jusqu'à il y a quelques années, il y avait cette entreprise, que je disais être devenue une espèce d'institution divine. Maintenant il y a toujours la force de l'entreprise mais il y a beaucoup plus de gens déçus, essayant de tirer les ficelles et de se comporter comme de pures individualités, ce qui affaiblit le lien social. Et naturellement cela ne donne pas des individus qui sont capables d'insuffler du dynamisme, se disant : «Je donne beaucoup mais l'entreprise me rend beaucoup ». On s'aperçoit qu'il commence à y avoir des interrogations sur la mondialisation, sur l'écologie et, même s'il n'y a pas une rénovation de l'action des syndicats parce qu'ils sont touchés comme les autres, il y a de plus en plus de gens qui essayent dans une certaine mesure de se demander s'il n'y a pas d'autres possibilités. De petites entreprises se créent avec du micro crédit, des associations, des jeunes entrepreneurs fonctionnent d'une autre manière. Pour l'instant, ce sont quelques gouttes d'eau, mais le grand moment du capitalisme capable de faire que les gens s'identifient profondément à l'entreprise est passé.

Alors que va-t-il arriver? Je ne sais pas encore, mais je ressens de plus en plus de retrait, de déception et, en même temps chez beaucoup de gens, le désir de s'organiser, de créer des lieux un peu à soi, des petites entreprises, des petits restaurants coopératifs, cela peut-être simplement pour se protéger eux-mêmes. Il y a des éléments d'économie solidaire qui apparaissent et qui me semblent être non pas une véritable 
alternative à l'heure actuelle, mais que l'on doit prendre en considération pour voir effectivement les évolutions futures.

Vous avez expliqué, au début de cet entretien, votre choix d'une orientation politique. Dans l'ouvrage, que vous avez écrit avec Claudine Haroche, La face obscure des démocraties modernes ${ }^{10}$ vous évoquez leur développement mortifère. Pouvons-nous espérer que les individus, là aussi, vont commencer à résister?

Oui, c'est pour cela que notre dernier chapitre s'intitule "penser, résister". Les gens sont toujours étonnés, quand ils me lisent, parce qu'ils trouvent toujours le côté noir de la chose et, ils sont toujours étonnés quand ils me connaissent, de voir que je suis un homme qui aime la vie, qui est souriant, dynamique. J'ai été frappé par la citation de Scott Fitzgerald qui disait «Il faut être certain que le monde est sans espoir et néanmoins être décidé à le changer». C'est vrai qu'il y a tout un mouvement intéressant qui montre que les choses ne sont pas jouées. Je pense au livre de Susan George Un autre monde est possible si... ${ }^{11}$, à celui de l'économiste indien Amartya Sen qui a eu le prix Nobel ${ }^{12}$. Je n'ai pas envie d'écouter les sirènes qui disent que cela va aller mieux bientôt. Je l'espère, je le dis. Mais, je me suis fixé comme tâche d'essayer de montrer où cela faisait mal de manière à ce que cela fasse moins mal. C'est pourquoi j'écris encore sur la violence et la haine. Peut-être qu'un jour je changerai. Mes amis aperçoivent un changement car je commence à rédiger des textes sur l'amour.

Selon vous, étudier la structuration des organisations implique de conjuguer Recherche et Intervention; vous en avez d'ailleurs parlé. Que diriez-vous aux chercheurs pour expliquer cet impératif méthodologique?

Je considère qu'il peut y avoir de très bons chercheurs pour qui la question ne se pose pas. Il peut y avoir de très bons chercheurs qui retracent l'évolution historique, des théoriciens de l'action. Je suis plus interrogatif sur le très bon chercheur dans le domaine des organisations. Attention! Je ne veux pas dire que cela ne soit pas possible, j'ai formé de nombreuses personnes à faire de la recherche dans les organisations. Mais je suis toujours gêné, et c'est ce que l'on peut dire aux chercheurs, par le fait que lorsque l'on fait une recherche trop distanciée par rapport aux organisations, on a une photographie de ce qui existe, mais on n'a pas la dynamique de ce qui peut exister. Si je relie recherche et intervention, c'est qu'il me semble que, dans toute organisation, il y a toujours des éléments dynamiques, des éléments de changement, et que je n'ai pas envie de les chosifier. Il ne faut pas oublier que dans une recherche, la plupart du temps les gens diront plus ce qui va mal que ce qui va bien. De toute façon, il y a toujours quelque chose qui me semble un peu biaisé mais surtout, ce qui me fait aller plus du côté de l'intervention, c'est que j'ai envie de saisir ce que j'étudie dans son côté vivant, dynamique. Je rappelle toujours les très belles phrases de Marcel Mauss, en conclusion de L'essai sur le don ${ }^{13}$ que je cite approximativement: "Je ne veux pas étudier les sociétés dans leur aspect inerte ou même cadavérique. Je veux étudier les sociétés dans leur physiologie, dans leur côté vivant et ce que je veux voir c'est le groupe tout entier et sa manière de vivre. " J'ai l'impression, quand on fait de simples recherches, qu'on finit toujours, même si on veut garder le côté vivant, par donner une image un peu statique de la réalité. Vous voyez, ce qui m'intéresse, c'est ce qui est en train de se tramer, les petits mouvements imperceptibles de la société. 
Actuellement quels sont les petits mouvements repérés par la psychosociologie et qu'elle pourrait étudier?

La psychosociologie s'intéresse à beaucoup plus de problèmes qu'auparavant. Entre la psychosociologie et la sociologie clinique, il n'y a plus beaucoup de différences. Elle s'intéresse aussi aux phénomènes des banlieues, aux systèmes ouverts, aux phénomènes organisationnels. Elle s'intéresse plus aux phénomènes d'environnement, à des phénomènes politiques. Et d'ailleurs, elle est moins sûre d'elle-même, elle est née dans le petit groupe, dans l'organisation, dans l'institution un peu solide, un peu fermée. Donc elle est moins sûre et, en même temps, je pense qu'elle va se renouveler dans la mesure où elle doit peut-être inventer de nouvelles méthodes. Je suis très frappé par des gens qui me semblent plus dynamiques que nous, par exemple, des psychosociologues italiens qui sont intervenus dans une région d'Italie, l'Émilie Romagne, pour apporter leur aide à la transformation complète du secteur sanitaire dans toute la province. Ils ont eu des actions très profondes. Ils ont été obligés d'inventer des méthodes très différentes, avec parfois des grandes réunions, des grands forums. Donc j'ai le sentiment qu'on est dans une phase de transition où il y a certainement beaucoup de choses à réinventer. Je ne pense pas être parmi ceux qui vont réinventer, mais je fais confiance aux jeunes générations de psychosociologues pour cela.

\section{Épilogue}

Le prochain livre, non pas celui que je dois finir, mais celui que je dois écrire, ce sera sur une philosophie de l'amour. Mes amis me disent : « Enfin ce sera l'Eugène positif qui apparaîtra ». Il apparaîtra peut-être un peu tard. Effectivement j'ai passé plus de temps à montrer les difficultés, l'œuvre de la pulsion de mort que de la pulsion de vie, mais cela a toujours été pour permettre aux gens de mieux se rendre compte des choses difficiles, mauvaises, horribles qui peuvent exister, de façon à ce qu'ils puissent s'en protéger. C'est noir, mais il y a toujours eu, malgré tout, un message d'espérance derrière cela. J'ai toujours été, en même temps, celui qui pense à la barbarie, mais aussi celui qui pense qu'il faut de l'amour, de la camaraderie, de l'amitié. Ce sont des éléments tout à fait fondamentaux pour les gens et puis pour vivre, vivre tout simplement.

\section{NOTES}

1. Paris, P.U.F.

2. Paris, P.U.F., 1992.

3. De la horde à l'État, essai de psychanalyse du lien social, Paris, Gallimard, 1983.

4. Avec Claudine Haroche, Paris, Érès, 2002.

5. J. Barus-Michel, Fl. Giust-Desprairies, L. Ridel, Crises, Approche psychosociale clinique, Paris, Desclée de Brouwer, 1996.

6. Paris, Seuil, 2005.

7. Paris, Flammarion, 2004.

8. La fatigue d'être soi. Dépression et société, Paris, Odile Jacob, 1998. 
9. Paris, Desclée de Brouwer, 1997.

10. Op.cit.

11. Paris, Fayard, 2002.

12. Un nouveau modèle économique. Développement, justice, liberté, Paris, Odile Jacob, 2000.

13. "Essai sur le don. Forme et raison de l'échange dans les sociétés archaïques ", in : Année sociologique, 1923-1924. 